\title{
Niosomes of Ascorbic Acid and $\alpha$-Tocopherol in the Cerebral Ischemia-Reperfusion Model in Male Rats
}

\author{
Jaleh Varshosaz, ${ }^{1}$ Somayeh Taymouri, ${ }^{2}$ Abbas Pardakhty, \\ Majid Asadi-Shekaari, ${ }^{3}$ and Abodolreza Babaee ${ }^{4}$ \\ ${ }^{1}$ Department of Pharmaceutics, School of Pharmacy and Novel Drug Delivery Systems Research Centre, \\ Isfahan University of Medical Sciences, P.O. Box 81745-359, Isfahan, Iran \\ ${ }^{2}$ Pharmaceutics Research Center, Neuropharmacology Institute, Kerman University of Medical Sciences, \\ P.O. Box 76175-493, Kerman, Iran \\ ${ }^{3}$ Neuroscience Research Center, Neuropharmacology Institute, Kerman University of Medical Sciences, \\ Kerman 7619813159, Iran \\ ${ }^{4}$ Anatomical Sciences Department, Afzali Pour Medical Faculty, Kerman University of Medical Sciences, \\ P.O. Box 76175-493, Kerman, Iran
}

Correspondence should be addressed to Abbas Pardakhty; abpardakhty@kmu.ac.ir

Received 22 January 2014; Accepted 26 June 2014; Published 28 August 2014

Academic Editor: Antonio Salgado

Copyright (C) 2014 Jaleh Varshosaz et al. This is an open access article distributed under the Creative Commons Attribution License, which permits unrestricted use, distribution, and reproduction in any medium, provided the original work is properly cited.

The objective of the present study was to prepare a stable $i v$ injectable formulation of ascorbic acid and $\alpha$-tocopherol in preventing the cerebral ischemia. Different niosomal formulations were prepared by Span and Tween mixed with cholesterol. The physicochemical characteristics of niosomal formulations were evaluated in vitro. For in vivo evaluation, the rats were made ischemic by middle cerebral artery occlusion model for $30 \mathrm{~min}$ and the selected formulation was used for determining its neuroprotective effect against cerebral ischemia. Neuronal damage was evaluated by optical microscopy and transmission electron microscopy. The encapsulation efficiency of ascorbic acid was increased to more than $84 \%$ by remote loading method. The cholesterol content of the niosomes, the hydrophilicity potential of the encapsulated compounds, and the preparation method of niosomes were the main factors affecting the mean volume diameter of the prepared vesicles. High physical stability of the niosomes prepared from Span 40 and Span 60 was demonstrated due to negligible size change of vesicles during 6 months storage at $4-8^{\circ} \mathrm{C}$. In vivo studies showed that $\mathrm{ST} 60 / \mathrm{Chol} 35: 35: 30$ niosomes had more neuroprotective effects against cerebral ischemic injuries in male rats than free ascorbic acid.

\section{Introduction}

Cerebral ischemia is the third leading cause of deaths in the United States [1]. In patient with acute cerebral ischemia, thrombolysis therapy could reduce death after ischemia [2]. Recombinant tissue plasminogen activator (r-TPA) is the only FDA approved drug which may be effective in 3 hours of symptom onset [3]. However, intracerebral hemorrhage (ICH) and mortality risk with r-TPA have led to development of alternative therapies [2]. Brain is a susceptible tissue to oxidative stress due to high level of free radicals, high amounts of unsaturated fatty acids, and fair protective antioxidant capacity in different parts [4]. It is reported that free radical oxygen has potential role in neural cell damage in ischemia-reperfusion disorders $[5,6]$. Exogenous antioxidant such as ascorbic acid (vitamin C), $\alpha$-tocopherol (vitamin E), and $\beta$-carotene can be effective on neuronal cell protection due to the effect of reactive oxygen species (ROS) on neuronal cell damages and fast consumption of endogenous scavenging antioxidants $[5,7]$. But their effectiveness depends on their ability for transporting through the blood brain barrier (BBB) [8]. It was reported that oral administration of $\alpha$ tocopherol supplementation despite making high plasma level could not increase $\alpha$-tocopherol level in ventricular cerebrospinal fluid even at high doses which could be related to limited passage from BBB [9]. On other hand, ascorbic 
acid (a hydrophilic molecule) cannot transport efficiently across the $\mathrm{BBB}$ due to its polarity and hydrophilicity [8]. To overcome these problems, different drug delivery systems such as dendrimers [10], liposomes [11], poly-butyl cyanoacrylate (PBCA) nanoparticles coated with polysorbate 80 [12], human serum albumin [13], solid lipid nanoparticles [14], and polymeric nanoparticles such as poly(lactide-coglycolic acid) ones [15] have been studied for increasing transport of different therapeutics across BBB. Liposomes containing ascorbic acid or $\alpha$-tocopherol were prepared by Sinha et al. [5]. The results were encouraging, but low chemical stability and thermo-liability of phospholipids, the main constituents of liposomes, made us develop niosomes of these two supplements which are more stable to chemicals and temperature compared to liposomes.

Noisomes are nonionic surfactant vesicles used as drug carrier similar to liposomes. The low cost, greater stability, and resultant ease of storage of nonionic surfactants have led to the development of these carriers as alternatives to liposomes [16]. There are some reports about the increased level of niosomal encapsulated drugs in brain of animals [17]. Increased uptake of methotrexate was observed after intravenous administration of methotrexate niosomal formulation in mice [18]. Dufes et al. [19] successfully used glucosebearing niosomes as a brain targeted delivery system for the vasoactive intestinal peptide (VIP). It was also reported that niosomal formulation of doxorubicin functionalized with the glucose-derivative $\mathrm{N}$-palmitoyl glucosamine was able to improve doxorubicin brain concentration in contrast to its commercial solution [20]. The successful ability of niosomal carriers to transport through $\mathrm{BBB}$ and the capability of niosomes in encapsulation of both hydrophilic and lipophilic compounds provided our rational for designing of niosomal formulation of antioxidant of vitamin $\mathrm{C}$ and $\mathrm{E}$ for brain delivery in preventing stroke. This carrier also provides the ability of $i v$ administration of lipid soluble molecules such as vitamin E.

Considering the more physical stability of niosomes than liposomes and regarding promising results of cerebral ischemia preventive effects reported for ascorbic acid and vitamin E liposomes [5], the main goal of our study was to prepare niosomal formulation of $\alpha$-tocopherol and ascorbic acid for enhanced brain delivery of these drugs in preventing neuronal cell damages during ischemia-reperfusion disorders. Different pharmaceutical parameters such as particle size, encapsulation efficiency, in vitro release of encapsulated materials, and stability of the designed formulations were evaluated. In addition, the neuroprotective effects of the designed formulations were investigated in an ischemiareperfusion model in male rats. To our knowledge, there is no report on the production and application of niosomal formulation of these two vitamins in prevention of ischemiareperfusion model in rats.

\section{Materials and Methods}

2.1. Materials. Ascorbic acid and $\alpha$-tocopherol were purchased from Merck Chemical Company (Germany). The
TABLE 1: Composition of different niosomal formulation containing ascorbic acid or $\alpha$-tocopherol.

\begin{tabular}{lccccc}
\hline $\begin{array}{l}\text { Formulation code } \\
\text { Molar ratio }\end{array}$ & Cholesterol & $\begin{array}{c}\text { Span } \\
40\end{array}$ & $\begin{array}{c}\text { Tween } \\
40\end{array}$ & $\begin{array}{c}\text { Span } \\
60\end{array}$ & $\begin{array}{c}\text { Tween } \\
60\end{array}$ \\
\hline ST40/Chol 35:35:30 & 30 & 35 & 35 & - & - \\
ST40/Chol 30:30:40 & 40 & 30 & 30 & - & - \\
ST40/Chol 25:25:50 & 50 & 25 & 25 & - & - \\
ST60/Chol 35:35:30 & 30 & - & - & 35 & 35 \\
ST60/Chol 30:30:40 & 40 & - & - & 30 & 30 \\
ST60/Chol 25:25:50 & 50 & - & - & 25 & 25 \\
\hline
\end{tabular}

nonionic surfactants used as vesicle-forming materials including polysorbate 40 (Tween 40), polysorbate 60 (Tween 60 ), sorbitan monopalmitate 40 (Span 40), sorbitan monostearate 60 (Span 60), and cholesterol ( $\mathrm{Chol}$ ) were purchased from Fluka Company (Switzerland). All organic solvents and the other chemicals were of analytical grade and obtained from Merck Chemical Company (Germany).

\subsection{Preparation of Drugs-Loaded Niosomes}

2.2.1. Conventional Film Hydration Method. Vesicular formulations containing ascorbic acid or $\alpha$-tocopherol were prepared by film hydration method [21]. The composition of different niosomal formulations is shown in Table 1. Briefly, niosomes of ascorbic acid were prepared by dissolving $400 \mu \mathrm{mol}$ of surfactants (equal molar percent of Tween/Span with the same hydrocarbon chain type and length)/Chol in chloroform in a round-bottomed flask. The organic solvent was evaporated under reduced pressure at $55^{\circ} \mathrm{C}$. The resultant thin lipid film produced on the inner wall of the flask was then hydrated with $10 \mathrm{~mL}$ of normal saline $0.9 \mathrm{w} / \mathrm{v} \%$ solution containing $2.5 \mathrm{mg} / \mathrm{mL}$ of ascorbic acid at $55^{\circ} \mathrm{C}$ for $30 \mathrm{~min}$. $\alpha$-Tocopherol niosomal formulations were prepared by the same amounts and types of surfactants/Chol and the same method as ascorbic acid but $20 \mathrm{mg}$ of $\alpha$-tocopherol was dissolved in the chloroform along with the other lipids in the round-bottomed flask. The compositions of the different formulations of vesicles are listed in Table 1.

2.2.2. Remote Loading Method. Ascorbic acid was loaded into preformed niosomes by modifying a remote-loading technique [22-24]. The composition of different niosomal formulations is shown in Table 1 . Briefly, $400 \mu \mathrm{mol}$ of surfactants and cholesterol was dissolved in chloroform in a roundbottomed flask. The organic solvent was evaporated under reduced pressure at $55^{\circ} \mathrm{C}$. The dried lipid film was hydrated with $5 \mathrm{~mL}$ calcium acetate $(200 \mathrm{mM})$. Nonentrapped calcium acetate was removed from the niosomal suspension by dialysis (molecular weight cutoff of $12 \mathrm{kDa}$ ) against dextrose $5 \mathrm{w} / \mathrm{v} \%$ for $2 \mathrm{~h}$. Then, $5 \mathrm{~mL}$ of the solution of ascorbic acid with concentration of $5 \mathrm{mg} / \mathrm{mL}$ was added to the prepared niosomal suspension and mixed in a rotating water bath for $20 \mathrm{~min}$. 
2.3. Vesicle Size Measurement. The particle size of different formulations was measured using a static laser light diffraction method by Malvern particle size analyzer (Malvern Instruments, MasterSizer 2000E, UK) $48 \mathrm{~h}$ after preparation.

2.4. Encapsulation Efficiency Determination. To separate nonentrapped $\alpha$-tocopherol or ascorbic acid from niosomes, the vesicular suspensions were centrifuged (National labnet, USA) at $25000 \mathrm{rpm}$ for $60 \mathrm{~min}$ at $4^{\circ} \mathrm{C}$ and washed twice with normal saline $0.9 \mathrm{w} / \mathrm{v} \%$. The amount of entrapped $\alpha$ tocopherol or ascorbic acid in the niosomes was analyzed by UV/visible spectrophotometer (Shimadzu 2100, Japan) at $\lambda_{\text {max }}$ of 246 and $290 \mathrm{~nm}$ for ascorbic acid and $\alpha$-tocopherol, respectively, after disrupting the niosomes by ethanol $96 \%$. The encapsulation efficiency percent of $\alpha$-tocopherol or ascorbic acid (EE \%) was determined from

$$
\mathrm{EE} \%=\left(\frac{C_{p}}{C_{T}}\right) \times 100,
$$

where $C_{p}$ is the active component concentration encapsulated in the niosomes and $C_{T}$ is the initial drug concentration added to formulation. Empty niosomes were used as blank.

2.5. In Vitro Drug Release Studies. Ascorbic acid release from various formulations was evaluated using dialysis method. The dialysis membrane (molecular weight cutoff of $12 \mathrm{kDa}$ ) that contained $4 \mathrm{~mL}$ of ascorbic acid formulation was placed in a glass flask filled with $40 \mathrm{~mL}$ of normal saline $0.9 \mathrm{w} / \mathrm{v} \%$ as receptor compartment. Temperature was maintained at $37 \pm 1^{\circ} \mathrm{C}$ by a circulating water bath. The medium in the receptor compartment was magnetically stirred at a rate of $100 \mathrm{rpm}$. Samples of the medium were withdrawn at fixed time intervals and replaced with an equal volume of fresh normal saline for $6 \mathrm{~h}$. The drug released concentration in the medium was quantified spectrophotometrically. In the case of lipid soluble material, $\alpha$-tocopherol, drug release was negligible in the mentioned period of release test due to the low partitioning characteristics of vitamin $\mathrm{E}$ in aqueous media and intercalating of this material in lipid bilayers.

2.6. Physical Stability of Vesicles. The vesicles were stored in glass vials in refrigerator $\left(4-8^{\circ} \mathrm{C}\right)$ for 6 months and the changes in vesicles diameter were determined by laser light scattering method. The changes in morphology of multilayered vesicles (MLVs) and also the separation of constituents were assessed by the optical microscope (HFX-DX, Nikon, Japan). No special precautions were taken to improve the stability of the vesicles.

\subsection{In Vivo Studies}

2.7.1. Pretreatment of Animals with Niosomes and Induction of Cerebral Ischemia. Male Wistar rats weighing 220-270 g were used for study. Animals had free access to food and water before and after surgical procedures. All animal studies were done in compliance with the ethics guidelines approved by the Kerman University of Medical Science (Kerman, Iran).
Rats were divided into 7 groups: group 1 for ascorbic acid niosomal formulation, group 2 for $\alpha$-tocopherol niosomal formulation, group 3 for free ascorbic acid (as $\alpha$-tocopherol was not water soluble there was no treated group with iv administration of free $\alpha$-tocopherol), group 4 for normal saline (negative control), group 5 for blank niosomes, group 6 for a mixture of $\alpha$-tocopherol and ascorbic acid niosomes in a ratio of $1: 1(\mathrm{w} / \mathrm{w})$, and group 7 for sham-operated rats. All animals received either ascorbic acid or $\alpha$-tocopherol with a dose of $8 \mathrm{mg} / \mathrm{kg}$ body weight which was injected into the tail vein of rats 2-3 h before cerebral ischemia [5]. Animals were anesthetized with chloral hydrate $(400 \mathrm{mg} / \mathrm{kg})$ and were made ischemic by middle cerebral artery occlusion (MCAO) for $30 \mathrm{~min}$ [25]. Under the neurosurgical microscope, the right common carotid artery (CCA) was exposed to a midline incision. After blocking all branches of the external carotid artery (ECA) and extra cranial branches of the internal carotid artery (ICA), a 4-0 nylon intraluminal suture was introduced into cervical ICA and advancing in intracranially to block blood flow into the middle cerebral artery (MCA). Following thirty minutes, suture was withdrawn and blood flow resumed. After recovery from the anesthesia, the animals were returned to their home cages. Two days after ischemia, the animals were tested for neurological examination. Neuronal damage was evaluated by optical microscopy and was estimated as a rate of the number of degenerated pyramidal neurons to that of both surviving and degenerated in three distinct areas of the cortex subfield in coronal sections for each animal [26]. The morphology of neurons was evaluated under transmission electron microscope (EM300, Philips, Holland).

\section{Results and Discussion}

3.1. Encapsulation Efficiency. Encapsulation efficiencies (EE) in all studied formulations are shown in Table 2. $\alpha$ Tocopherol had often higher EE\% than ascorbic acid, which is clearly due to its hydrophobic nature and its intercalating in lipophilic core of surfactant bilayers [27]. Increasing the amount of Chol from 30 to 50 mole percent reduced the EE\% of $\alpha$-tocopherol in both types of studied surfactants which was due to the competition between $\alpha$-tocopherol and Chol molecules. Remote-loading procedure by using an ammonium sulfate gradient method [28] and complexation with arylsulfonates are two ways reported for intravesicular precipitate and enhancement of drug retention [29]. In our study, higher encapsulation efficiency of ascorbic acid was achieved by the remote-loading procedure by using calcium acetate gradient method (Table 2) compared to the other loading method $(P<0.05)$. It was supposed that during remote loading ascorbic acid diffused in uncharged form via niosome membrane due to calcium acetate and $\mathrm{pH}$ trans-membrane gradient. There, it loses its proton, becomes negatively charged, and forms a poorly soluble ascorbate calcium salt, which precipitates in the intraniosomal aqueous phase. This method also improved encapsulation efficiency of ciprofloxacin [30], diclofenac, insulin, and fluorescein isothiocyanate labeled insulin [22] in liposomes and luteinizing 
TABLE 2: Encapsulation efficiency of different formulations containing vitamin $\mathrm{C}$ and vitamin $\mathrm{E}$.

\begin{tabular}{|c|c|c|c|}
\hline \multirow{2}{*}{ Niosomal formulations } & \multicolumn{2}{|c|}{ Vitamin C encapsulation efficiency \% } & \multirow{2}{*}{$\begin{array}{c}\text { Vitamin E encapsulation efficiency \% } \\
\text { Film hydration }\end{array}$} \\
\hline & Remote loading & Film hydration & \\
\hline ST40/Chol $35: 35: 30$ & $55.24 \pm 0.17$ & $7 \pm 3.56$ & $88.136 \pm 4.90$ \\
\hline ST40/Chol $30: 30: 40$ & $70.54 \pm 0.14$ & $7.66 \pm 1.2$ & $83.51 \pm 7.60$ \\
\hline ST40/Chol $25: 25: 50$ & $66.68 \pm 2.47$ & $8.53 \pm 0.12$ & $65.30 \pm 12.13$ \\
\hline ST60/Chol $35: 35: 30$ & $79.63 \pm 0.56$ & $10 \pm 0.634$ & $91.5 \pm 3.54$ \\
\hline ST60/Chol $30: 30: 40$ & $85.94 \pm 5.47$ & $9.11 \pm 1.54$ & $82.17 \pm 18.63$ \\
\hline ST60/Chol $25: 25: 50$ & $48.36 \pm 9.41$ & $9.63 \pm 2.34$ & $56.85 \pm 9.27$ \\
\hline
\end{tabular}

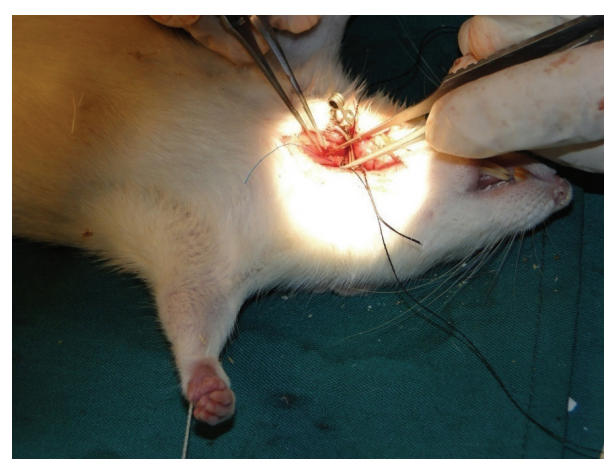

(a)

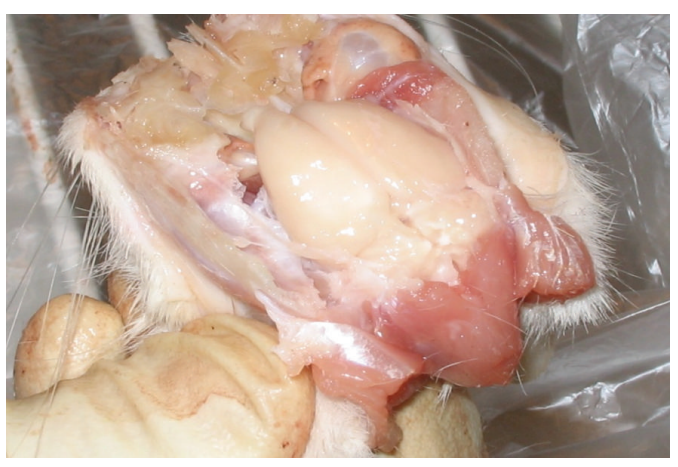

(b)

FIGURE 1: Surgical technique of middle cerebral artery occlusion in rat.

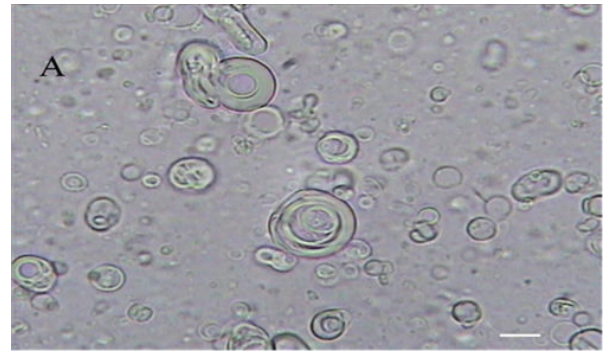

(a)

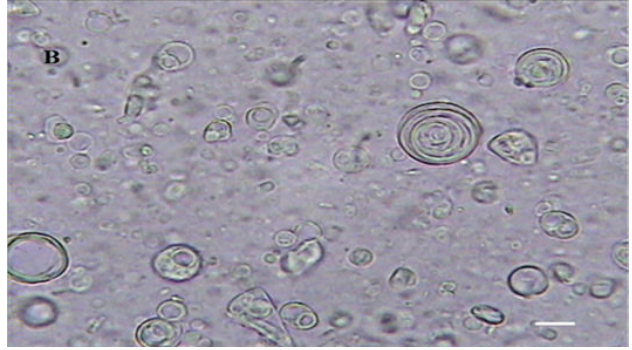

(b)

FIgURE 2: Optical micrographs of ascorbic acid or $\alpha$-tocopherol containing niosomes ( $\times 400$ magnification). Niosomes were composed of (a) Span Tween 40/cholesterol (30:30:40 mole\%) ascorbic acid, (b) Span Tween 60/cholesterol (35:35:30 mole\%) $\alpha$-tocopherol. (Scale bar: $5 \mu \mathrm{m})$.

hormone releasing hormone niosomes [31]. Increasing the molar ratio of Chol from 30 to 40 increased the EE\% of ascorbic acid significantly $(P<0.05)$ (Table 2$)$. This can be related to increasing the rigidity of the bilayers following Chol content enhancement which is parallel to gel transition temperature abolishment following Chol incorporation in lipid bilayers [32].

3.2. Morphology and Size Distribution of Vesicles. All used nonionic surfactant compositions formed niosomes in the presence of cholesterol. Figure 2 demonstrates the formation of niosomes in different formulations. Morphologically, the formulated niosomes were frequently as round MLVs as depicted in Figure 1. Obviously, this was predictable as film hydration method usually produces MLVs.
The mean volume diameters $\left(d_{v}\right)$ of the prepared nonionic surfactant vesicles with different compositions are presented in Tables 3 and 4. From data presented in these tables, it is obvious that, following the increase of cholesterol molar percent, the $d_{v}$ of neutral niosomes prepared by film hydration or remote loading methods was increased. Moazeni et al. [33] also reported the forming of the greater ciprofloxacin niosomes after raising the cholesterol content from 30 to 40 and 50 molar percent. The integration of the drug also has a significant effect on the particle size of the vesicles. It was revealed that the incorporation of vitamin $\mathrm{C}$ or vitamin $\mathrm{E}$ in all formulations led to size reduction compared to empty niosomes $(P<0.05)$ (the results of empty vesicles were not shown here). In a similar manner, Junyaprasert et al. [34] reporeted a slight size reduction in niosomal suspensions 
TABLE 3: Mean volume diameter of ascorbic acid loaded niosomes prepared by film hydration method after $48 \mathrm{~h}$ and remote loading method at different time intervals after preparation and storage at $4^{\circ} \mathrm{C}$ (mean $\left.\pm \mathrm{SD}, n=3\right)$.

\begin{tabular}{lccccc}
\hline Niosomal formulations & $\begin{array}{c}\text { Film hydration } \\
d_{v}(\mu \mathrm{m}) \pm \mathrm{SD}\end{array}$ & \multicolumn{3}{c}{$\begin{array}{c}\text { Remote loading } \\
d_{v}(\mu \mathrm{m}) \pm \mathrm{SD}\end{array}$} \\
& $48 \mathrm{~h}$ & $48 \mathrm{~h}$ & $1 \mathrm{month}$ & $2 \mathrm{months}$ & $6 \mathrm{months}$ \\
\hline ST40/Chol 35:35:30 & $5.06 \pm 0.13$ & $9.10 \pm 0.84$ & $7.80 \pm 0.11$ & $7.83 \pm 0.429$ & $6.87 \pm 0.106$ \\
ST40/Chol 30:30:40 & $7.55 \pm 0.38$ & $9.14 \pm 0.25$ & $8.25 \pm 0.15$ & $8.00 \pm 0.21$ & $7.921 \pm 0.354$ \\
ST40/Chol 25 $: 25: 50$ & $8.73 \pm 0.05$ & $8.87 \pm 0.13$ & $8.48 \pm 0.08$ & $8.38 \pm 0.130$ & $8.03 \pm 0.159$ \\
ST60/Chol 35:35:30 & $6.95 \pm 0.07$ & $7.06 \pm 0.13$ & $7.15 \pm 0.64$ & $6.03 \pm 0.098$ & $5.225 \pm 0.057$ \\
ST60/Chol 30:30:40 & $9.35 \pm 0.59$ & $8.78 \pm 0.29$ & $7.87 \pm 0.13$ & $7.49 \pm 0.50$ & $7.069 \pm 0.168$ \\
ST60/Chol 25:25:50 & $9.04 \pm 0.34$ & $9.06 \pm 0.14$ & $9.48 \pm 0.81$ & $8.98 \pm 0.054$ & $8.717 \pm 0.101$ \\
\hline
\end{tabular}

TABLE 4: Mean volume diameter of $\alpha$-tocopherol loaded niosomes at different time intervals after preparation and storage at $4^{\circ} \mathrm{C}(\mathrm{mean} \pm$ $\mathrm{SD}, n=3)$

\begin{tabular}{lcccc}
\hline Niosomal formulations & \multicolumn{3}{c}{$d_{v}(\mu \mathrm{m}) \pm \mathrm{SD}$} \\
& $48 \mathrm{~h}$ & $1 \mathrm{month}$ & 2 months & 6 months \\
\hline ST40/Chol 35:35:30 & $7.60 \pm 0.40$ & $8.11 \pm 0.38$ & $7.15 \pm 0.32$ & $7.23 \pm 0.25$ \\
ST40/Chol 30:30:40 & $8.14 \pm 0.36$ & $8.07 \pm 0.29$ & $9.22 \pm 0.14$ & $9.17 \pm 0.22$ \\
ST40/Chol 25:25:50 & $9.86 \pm 0.12$ & $9.61 \pm 0.08$ & $5.85 \pm 0.27$ & $9.20 \pm 0.19$ \\
ST60/Chol 35:35:30 & $6.42 \pm 0.08$ & $6.02 \pm 0.17$ & $8.48 \pm 0.49$ & $6.11 \pm 0.24$ \\
ST60/Chol 30:30:40 & $9.36 \pm 0.47$ & $7.19 \pm 0.29$ & $7.47 \pm 0.1$ & $10.22 \pm 0.27$ \\
ST60/Chol 25:25:50 & $7.67 \pm 0.07$ & $8.47 \pm 0.09$ & & $7.42 \pm 0.10$ \\
\hline
\end{tabular}

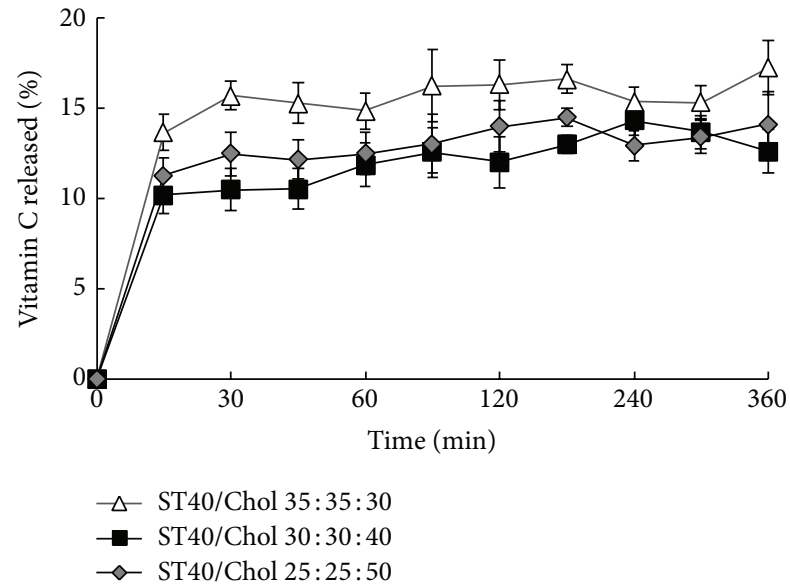

FIGURE 3: Release profiles of ascorbic acid from niosomes composed of Span-Tween $40 /$ Cholesterol in normal saline at $37^{\circ} \mathrm{C}($ mean $\pm \mathrm{SD}$, $n=3)$.

composed of Span 60/Chol in citrate buffer ( $\mathrm{pH}$ 5) following the encapsulation of salicylic acid. Another important factor that affected the particle size of vesicles was the method of preparation. The mentioned niosomes prepared by film hydration technique were significantly smaller than remote loading vesicles $(P<0.05)$. In ascorbic acid, niosomal formulations prepared from Span/Tween 40 and at the low levels of Chol (30 and 40 molar ratios), the vesicles volume diameter was more influenced by the preparation method as depicted in Table 3. The difference in dimensions of Span/Tween 60 niosomes prepared by two methods is also

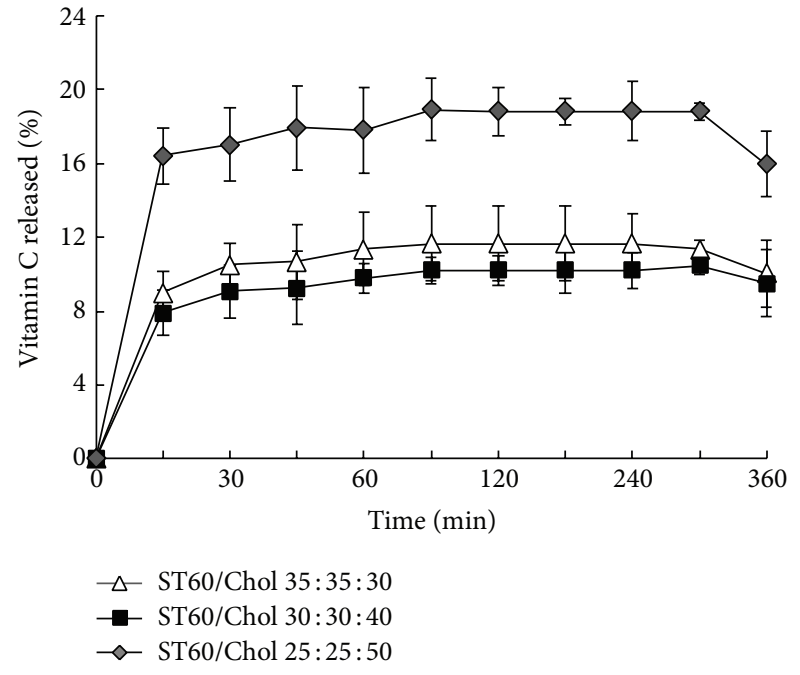

FIGURE 4: Release profiles of ascorbic acid from niosomes composed of Span-Tween $60 /$ Cholesterol in normal saline at $37^{\circ} \mathrm{C}($ mean $\pm \mathrm{SD}$, $n=3)$.

seen in Table 2 but with less extent. More rigidity of the bilayers composed of stearyl alkyl chain $\left(C_{18}\right)$ in Span/Tween 60 niosomes in comparison to palmityl alkyl chain $\left(C_{16}\right)$ of Span/Tween 40 may explain this finding.

3.3. In Vitro Release Studies. The type of entrapped molecule, vesicle lamellarity, and presence or absence of the charging agents must be considered in drug permeation studies in vesicular systems. The chemical structure of bilayer forming 


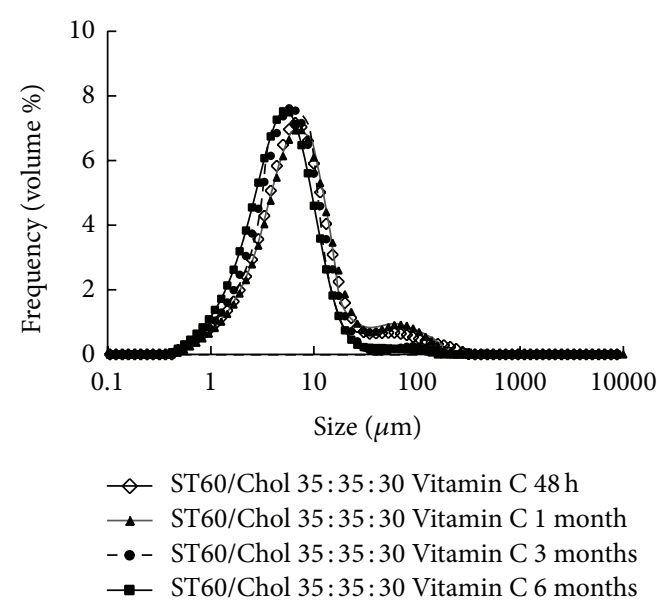

(a)

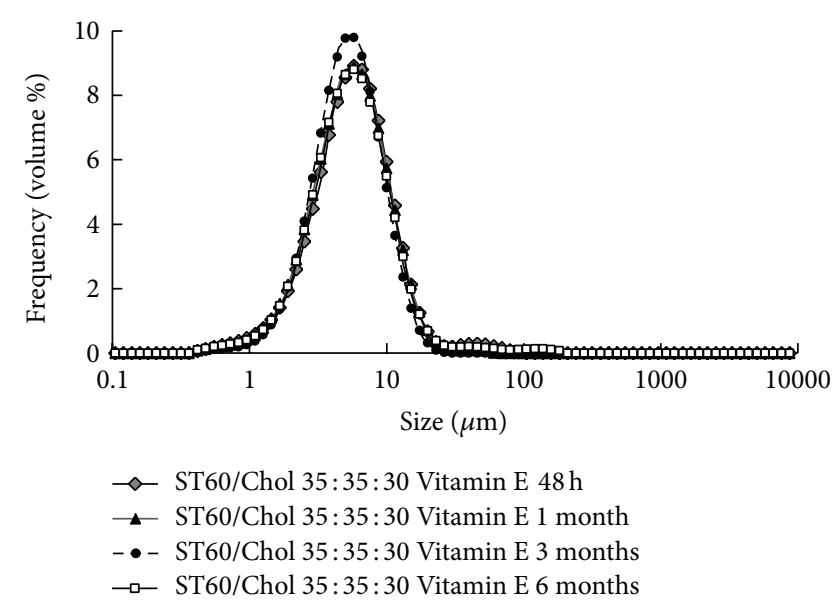

(b)

Figure 5: The size distribution changes of niosomes composed of (a) Span-Tween 60/Cholesterol $35: 35: 30$ contining vitamin C, (b) SpanTween 60/Cholesterol $35: 35: 30$ contining vitamin E during storage at $4^{\circ} \mathrm{C}$ as an indicator of physical stability of vesicles.

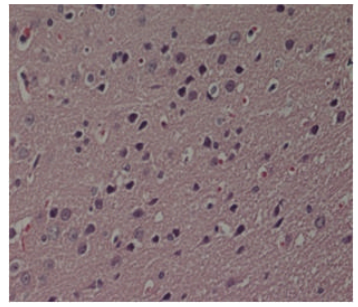

(a)

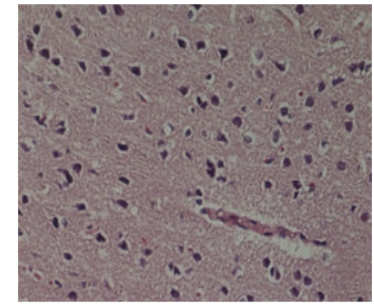

(b)

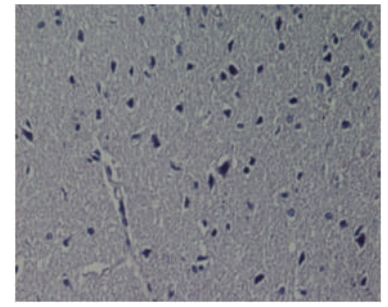

(c)

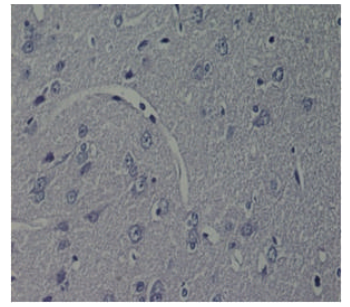

(d)

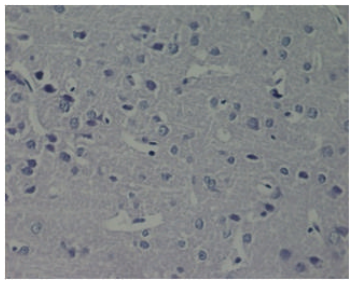

(e)

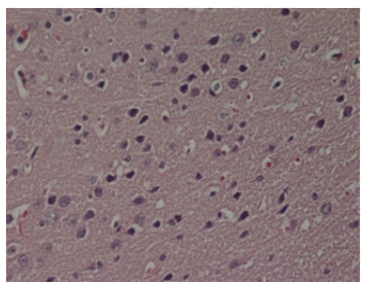

(f)

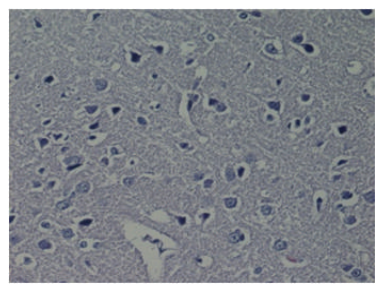

(g)

FIGURE 6: Histological changes in brain cortex from (a) sham-operated, (b) empty noisome, (c) saline-treated, (d) free ascorbic acid, (e) ascorbic noisome, (f) $\alpha$-tocopherol noisome, and (g) niosome formulation with a mixture of $\alpha$-tocopherol and ascorbic acid in a ratio of $1: 1$ $(\mathrm{w} / \mathrm{w})$ treated rats. As shown in the figure, morphology of the neurons in sham-operated group is intact. Severe degenerative changes (dark nucleus and shrunken cytoplasm) are present in the saline-treated and empty noisome groups. These changes were less in the other groups, particularly in the niosomal formulation with a mixture of $\alpha$-tocopherol and ascorbic acid. (Magnification $\times 400$ ).

lipids has clear effect on drug efflux from vesicles as well. In many cases, the drug release profile from niosomal systems are biphasic such as caffeine [35] and insulin [32] indicating a rapid desorption and a slower diffusion of entrapped drug though bilayer phases. In the present study, similar biphasic release was observed (Figures 3 and 4 ). The rapid initial phase may be related to desorption of drug from the surface of niosomes. After the initial burst release, a constant ascorbic acid release was observed during $360 \mathrm{~min}$ which was due to diffusion of ascorbic acid from lipid bilayer. According to this result, it took time for ascorbate ion to be released from multiple bilayers of niosomal vesicles that were stabilized by cholesterol. Interestingly, the overall release amount of ascorbic acid is adjustable with EE\% of drug in which more $\mathrm{EE} \%$ led to less drug release.

3.4. Stability Studies. Tables 3 and 4 exhibit the change in particle size is as a major indicator for niosomes stability during storage at $4^{\circ} \mathrm{C}$ for 6 months. In the present study, high vesicular stability was observed as depicted in Figure 5. Higher stability in vesicular structure of formulations with more cholestrol content was seen $(P<0.05)$ (Tables 2 and 3 ). The role of cholestrol in increasing membrane stability was reported perviously [33]. On the other hand, relatively large diameter of all prepared niosomes $(>5 \mu \mathrm{m})$ maybe 


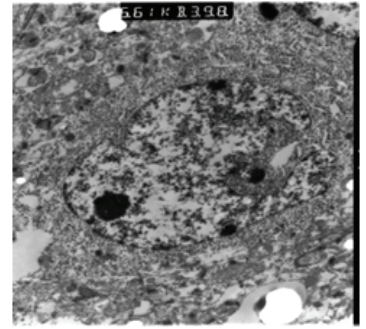

(a)

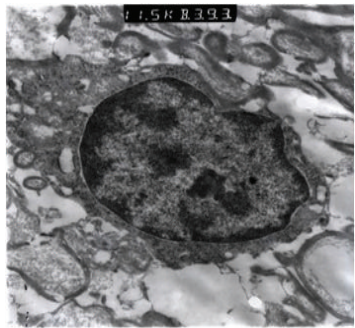

(b)

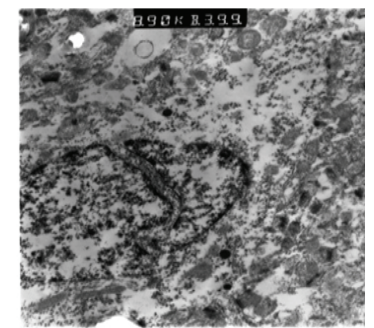

(c)

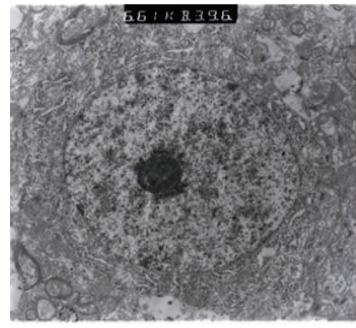

(d)

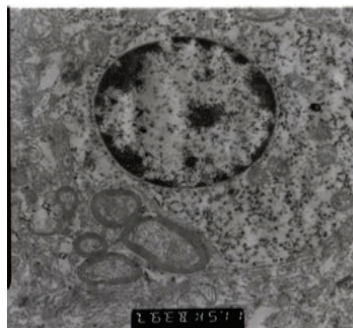

(e)

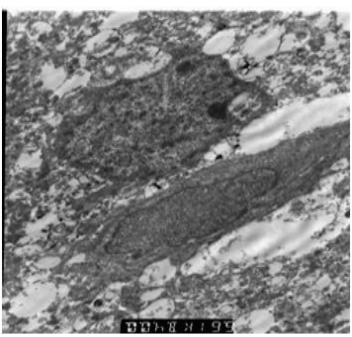

(f)

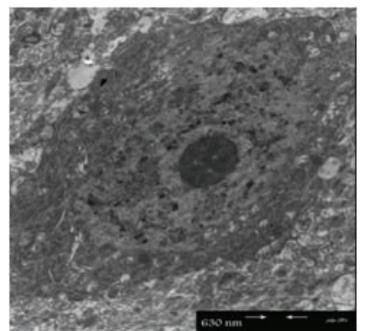

(g)

FiguRE 7: Electron micrograph of cortical neurons from (a) sham-operated, (b) empty noisome, (c) saline treated, (d) free ascorbic acid, (e) ascorbic noisome, (f) $\alpha$-tocopherol noisome, and (g) niosomal formulation with a mixture of $\alpha$-tocopherol and ascorbic acid treated rats. As shown in the micrograph, the normal ultrastructure of cortical neuron is visible in sham-operated group. Ischemia-reperfusion resulted in severe degenerative changes including chromatin aggregation, organelles swelling of the neurons in the saline-treated and empty niosome groups. Meanwhile, the whole ultrastructure of cortical neurons was maintained in the other groups mostly in the niosome formulation with a mixture of $\alpha$-tocopherol and ascorbic acid. (Scale bar: $630 \mathrm{~nm}$ ).

another reason for observed vesicular stability during 6month storage at refrigerator temperature because smaller vesicles are thermodynamically unstable [33]. The last reason of our niosomal stability could be related to gel state nature of Span/Tween 40 and Span/Tween 60 bilayers which are less sensitive to temperature fluctuation and less desire for bilayer fusion. In some cases such as Span/Tween 40/Chol/vitamin $\mathrm{E}$ (50 molar ratio of $\mathrm{Chol}$ ), a slight decrease in mean volume diameter of vesicles was observed (Tables 3 and 4). Similar results were observed in formulations prepared from Brij 52 and cholesterol [32]. To explain this observation, two theories are suggested [36]: (i) the ions adsorption to the bilayer originates a change in the head groups charge and, as a consequence, the bilayer changes its curvature due to the electric interactions which in turn causes changing in their size and (ii) the concentration gradient at both membrane sides generates an osmotic force due to the membrane impermeability to some ions, the vesicles react sending off water, decreasing their size.

3.5. Animal Studies. According to the obtained data in light microscopy study (Figure 6), the morphology of neurons in sham-operated group was normal but the most of the pyramidal neurons in saline-treated and blank niosome groups showed severe degenerative changes including eosinophilic and shrunk cytoplasm with extensively dark picnotic nuclei. In experimental groups that received the drug, the severity of degenerative changes in cytoplasm and nucleus were significantly less compared to saline-treated groups and groups that received blank niosome $(P<0.05)$.
In addition, encapsulated niosomal formulations especially the mixture of $\alpha$-tocopherol and ascorbic acid niosomal formulation $(1: 1 \mathrm{w} / \mathrm{w} 4 \mathrm{mg} / \mathrm{kg})$ showed more positive effects in cerebral-induced injuries (Figure 6). In experimental groups receiving ascorbic acid, loaded niosome and a combination of ascorbic acid and $\alpha$-tocopherol niosomal formulation, the mean number of degenerated neurons were significantly smaller than free ascorbic acid $(P<0.05)$. However, the difference between the two niosomal groups were not statistically significant $(P>0.05)$.

For confirmation of our results in light microscopy, we also studied the ultrastructure of the pyramidal neurons in the cortex of male rats using transmission electron microscope (TEM).

Morphology of cortical neurons in sham-operated group was intact (Figure 7). Some degenerative changes including organelles swelling, chromatin aggregation, and darkening of nucleus were observed in saline-treated and blank noisome groups. Treatment with prepared drugs preserved the ultrastructure of the most cortical neurons especially the mixture of $\alpha$-tocopherol and ascorbic acid niosomal formulation $(1: 1 \mathrm{w} / \mathrm{w} 4 \mathrm{mg} / \mathrm{kg})$ (Figure 7). In other words, the electron microscopy results confirmed the data obtained by light microscopy.

\section{Conclusions}

To compensate the problems related to transport of natural antioxidants in brain ischemia due to stroke, two different compounds of $\alpha$-tocopherol and ascorbic acid were 
formulated in niosomes composed of sorbitane esters and their ethoxylated derivatives. The cholesterol content and the hydrophilicity potential of encapsulated compounds were the main factors affecting the mean volume diameter of the prepared vesicles. Furthermore, in ascorbic acid niosomes, the method of niosome preparation had obvious effect on the mean diameter of vesicles. High physical stability of gel state niosomes caused negligible size change during 6-month storage at $4-8^{\circ} \mathrm{C}$.

In vivo results showed that although there was no significant difference between the mean number of degenerated neurons in the group received ascorbic acid niosomes with those treated with a mixture of ascorbic acid and $\alpha$-tocopherol niosomes and the groups treated with $\alpha$ tocopherol niosomes alone $(P>0.05)$ but, it was significantly higher in the group receiving free ascorbic compared to the mixed niosomes. In other words, the effectiveness of the formulated new drug delivery system in protection of cerebral tissue against elevation in oxygen free radical concentration during cerebral ischemia-reperfusion course was more than the free ascorbic acid. In addition, the designed niosomal formulation provides a suitable possible way for $i v$ administration of a water insoluble drug like $\alpha$-tocopherol.

\section{Conflict of Interests}

The authors declare that there is no conflict of interests regarding the publication of this paper.

\section{References}

[1] T. Rhim, D. Y. Lee, and M. Lee, "Drug delivery systems for the treatment of ischemic stroke," Pharmaceutical Research, vol. 30, pp. 2429-2444, 2013.

[2] . Wardlaw J, G. Zoppo, T. Yamaguchi et al., Thrombolysis for Acute Ischaemic Stroke, Wiely, 2009.

[3] A. Hicks and J. Jolkkonen, "Challenges and possibilities of intravascular cell therapy in stroke," Acta Neurobiologiae Experimentalis, vol. 69, no. 1, pp. 1-11, 2009.

[4] I. Margaill, M. Plotkine, and D. Lerouet, "Antioxidant strategies in the treatment of stroke," Free Radical Biology and Medicine, vol. 39, no. 4, pp. 429-443, 2005.

[5] J. Sinha, N. Das, and M. K. Basu, "Liposomal antioxidants in combating ischemia-reperfusion injury in rat brain," Biomedicine and Pharmacotherapy, vol. 55, no. 5, pp. 264-271, 2001.

[6] T. Sugawara, M. Fujimura, N. Noshita et al., "Neuronal death/survival signaling pathways in cerebral ischemia," NeuroRx, vol. 1, no. 1, pp. 17-25, 2004.

[7] S. Sarkar and N. Das, "Mannosylated liposomal flavonoid in combating age-related ischemia-reperfusion induced oxidative damage in rat brain," Mechanisms of Ageing and Development, vol. 127, no. 4, pp. 391-397, 2006.

[8] J. Huang, D. B. Agus, C. J. Winfree et al., "Dehydroascorbic acid, a blood-brain barrier transportable form of vitamin C, mediates potent cerebroprotection in experimental stroke," Proceedings of the National Academy of Sciences of the United States of America, vol. 98, no. 20, pp. 11720-11724, 2001.

[9] E. J. Pappert, C. C. Tangney, C. G. Goetz et al., "Alphatocopherol in the ventricular cerebrospinal fluid of Parkinson's disease patients: dose-response study and correlations with plasma levels," Neurology, vol. 47, no. 4, pp. 1037-1042, 1996.

[10] A. J. Salgado, J. M. Oliveira, R. P. Pirraco et al., "Carboxymethylchitosan/poly(amidoamine) dendrimer nanoparticles in central nervous systems-regenerative medicine: effects on neuron/glial cell viability and internalization efficiency," Macromolecular Bioscience, vol. 10, no. 10, pp. 1130-1140, 2010.

[11] P. J. Gaillard, C. C. M. Appeldoorn, J. Rip et al., "Enhanced brain delivery of liposomal methylprednisolone improved therapeutic efficacy in a model of neuroinflammation," Journal of Controlled Release, vol. 164, no. 3, pp. 364-369, 2012.

[12] J. Kreuter, "Nanoparticulate systems for brain delivery of drugs," Advanced Drug Delivery Reviews, vol. 47, no. 1, pp. 65-81, 2001.

[13] K. Michaelis, M. M. Hoffmann, S. Dreis et al., "Covalent linkage of apolipoprotein E to albumin nanoparticles strongly enhances drug transport into the brain," Journal of Pharmacology and Experimental Therapeutics, vol. 317, no. 3, pp. 1246-1253, 2006.

[14] P. Blasi, S. Giovagnoli, A. Schoubben, M. Ricci, and C. Rossi, "Solid lipid nanoparticles for targeted brain drug delivery," Advanced Drug Delivery Reviews, vol. 59, no. 6, pp. 454-477, 2007.

[15] S. Gelperina, O. Maksimenko, A. Khalansky et al., "Drug delivery to the brain using surfactant-coated poly(lactide-coglycolide) nanoparticles: Influence of the formulation parameters," European Journal of Pharmaceutics and Biopharmaceutics, vol. 74, no. 2, pp. 157-163, 2010.

[16] R. Rajera, K. Nagpal, S. K. Singh, and D. N. Mishra, "Niosomes: a controlled and novel drug delivery system," Biological and Pharmaceutical Bulletin, vol. 34, no. 7, pp. 945-953, 2011.

[17] D. Paolino, D. Cosco, R. Molinaro, C. Celia, and M. Fresta, "Supramolecular devices to improve the treatment of brain diseases," Drug Discovery Today, vol. 16, no. 7-8, pp. 311-324, 2011.

[18] M. N. Azmin, A. T. Florence, R. M. Handjani-Vila, J. F. Stuart, G. Vanlerberghe, and J. S. Whittaker, "The effect of non-ionic surfactant vesicle (niosome) entrapment on the absorption and distribution of methotrexate in mice," Journal of Pharmacy and Pharmacology, vol. 37, no. 4, pp. 237-242, 1985.

[19] C. Dufes, F. Gaillard, I. F. Uchegbu, A. G. Schätzlein, J. Olivier, and J. Muller, "Glucose-targeted niosomes deliver vasoactive intestinal peptide (VIP) to the brain," International Journal of Pharmaceutics, vol. 285, no. 1-2, pp. 77-85, 2004.

[20] M. Bragagni, N. Mennini, C. Ghelardini, and P. Mura, "Development and characterization of niosomal formulations of doxorubicin aimed at brain targeting," Journal of Pharmacy and Pharmaceutical Sciences, vol. 15, no. 1, pp. 184-196, 2012.

[21] A. J. Baillie, A. T. Florence, L. R. Hume, G. T. Muirhead, and A. Rogerson, "The preparation and properties of niosomesnon-ionic surfactant vesicles," The Journal of Pharmacy and Pharmacology, vol. 37, no. 12, pp. 863-868, 1985.

[22] S. H. Hwang, Y. Maitani, X. R. Qi, K. Takayama, and T. Nagai, "Remote loading of diclofenac, insulin and fluorescein isothiocyanate labeled insulin into liposomes by $\mathrm{pH}$ and acetate gradient methods," International Journal of Pharmaceutics, vol. 179, no. 1, pp. 85-95, 1999.

[23] S. Clerc and Y. Barenholz, "Loading of amphipathic weak acids into liposomes in response to transmembrane calcium acetate gradients," Biochimica et Biophysica Acta-Biomembranes, vol. 1240, no. 2, pp. 257-265, 1995.

[24] Y. Avnir, R. Ulmansky, V. Wasserman et al., "Amphipathic weak acid glucocorticoid prodrugs remote-loaded into sterically 
stabilized nanoliposomes evaluated in arthritic rats and in a Beagle dog: a novel approach to treating autoimmune arthritis," Arthritis \& Rheumatism, vol. 58, no. 1, pp. 119-129, 2008.

[25] E. Z. Longa, P. R. Weinstein, S. Carlson, and R. Cummins, "Reversible middle cerebral artery occlusion without craniectomy in rats," Stroke, vol. 20, no. 1, pp. 84-91, 1989.

[26] A. S. Majid, P. Marzieh, D. Shahriar, S. K. Zahed, and K. T. Pari, "Neuroprotective effects of aqueous date fruit extract on focal cerebral ischemia in rats," Pakistan Journal of Medical Sciences, vol. 24, no. 5, pp. 661-665, 2008.

[27] M. Gonnet, L. Lethuaut, and F. Boury, "New trends in encapsulation of liposoluble vitamins," Journal of Controlled Release, vol. 146, no. 3, pp. 276-290, 2010.

[28] J. Conley, H. Yang, T. Wilson et al., "Aerosol delivery of liposome-encapsulated ciprofloxacin: aerosol characterization and efficacy against Francisella tularensis infection in mice," Antimicrobial Agents and Chemotherapy, vol. 41, no. 6, pp. 12881292, 1997.

[29] I. V. Zhigaltsev, N. Maurer, K. Edwards, G. Karlsson, and P. R. Cullis, "Formation of drug-arylsulfonate complexes inside liposomes: a novel approach to improve drug retention," Journal of Controlled Release, vol. 110, no. 2, pp. 378-386, 2006.

[30] Y.K. Oh, D. E. Nix, and R. M. Straubinger, "Formulation and efficacy of liposome-encapsulated antibiotics for therapy of intracellular Mycobacterium avium infection," Antimicrobial Agents and Chemotherapy, vol. 39, no. 9, pp. 2104-2111, 1995.

[31] P. Arunothayanun, J. A. Turton, I. F. Uchegbu, and A. T. Florence, "Preparation and in vitro/in vivo evaluation of luteinizing hormone releasing hormone (LHRH)-loaded polyhedral and spherical/tubular niosomes," Journal of Pharmaceutical Sciences, vol. 88, no. 1, pp. 34-38, 1999.

[32] A. Pardakhty, J. Varshosaz, and A. Rouholamini, "In vitro study of polyoxyethylene alkyl ether niosomes for delivery of insulin," International Journal of Pharmaceutics, vol. 328, no. 2, pp. 130141, 2007.

[33] E. Moazeni, K. Gilani, F. Sotoudegan et al., "Formulation and in vitro evaluation of ciprofloxacin containing niosomes for pulmonary delivery," Journal of Microencapsulation, vol. 27, no. 7, pp. 618-627, 2010.

[34] V. B. Junyaprasert, V. Teeranachaideekul, and T. Supaperm, "Effect of charged and non-ionic membrane additives on physicochemical properties and stability of niosomes," AAPS PharmSciTech, vol. 9, no. 3, pp. 851-859, 2008.

[35] P. Khazaeli, A. Pardakhty, and H. Shoorabi, "Caffeine-loaded niosomes: characterization and in vitro release studies," Drug Delivery, vol. 14, no. 7, pp. 447-452, 2007.

[36] L. di Marzio, C. Marianecci, M. Petrone, F. Rinaldi, and M. Carafa, "Novel pH-sensitive non-ionic surfactant vesicles: comparison between Tween 21 and Tween 20," Colloids and Surfaces B: Biointerfaces, vol. 82, no. 1, pp. 18-24, 2011. 

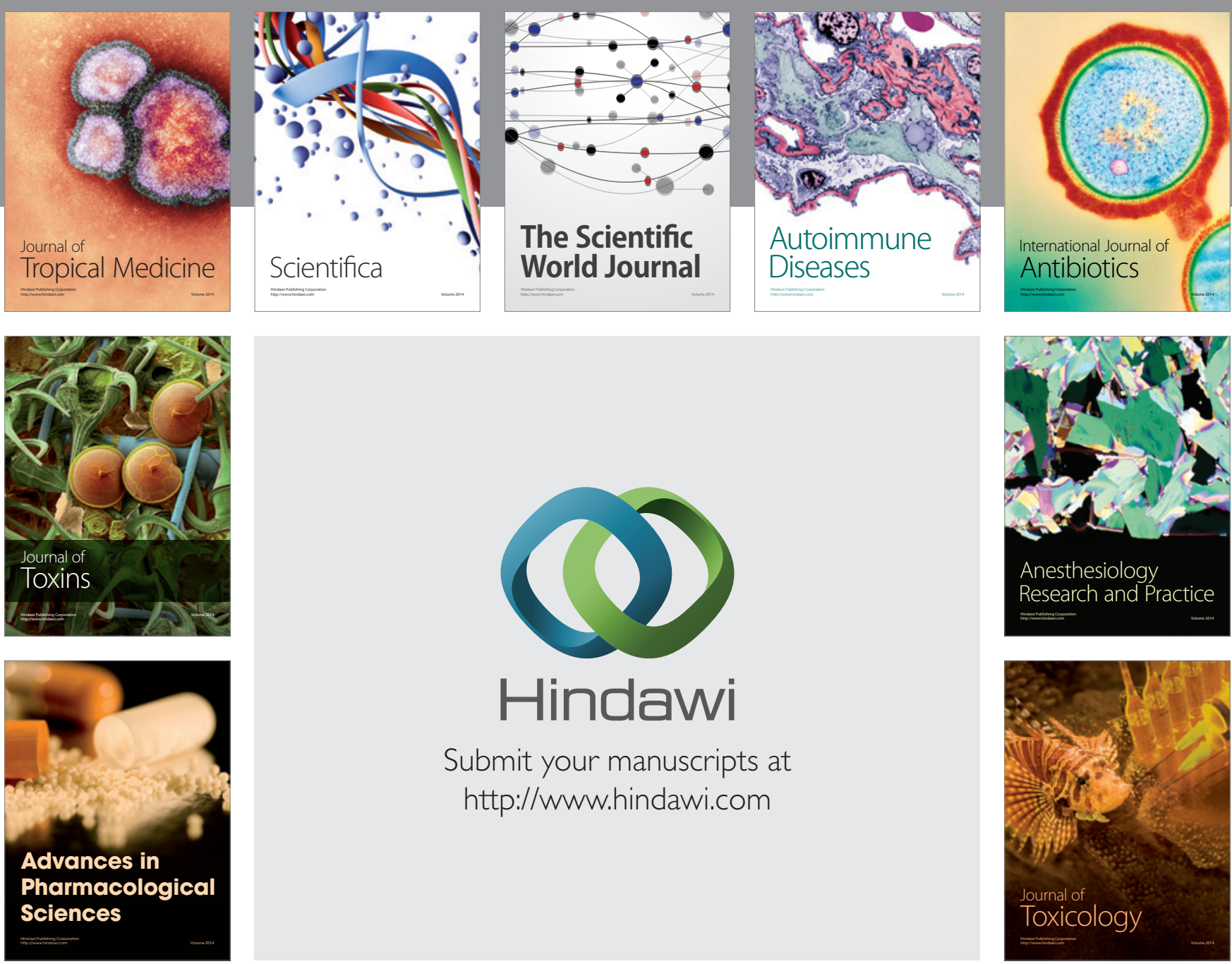

\section{Hindawi}

Submit your manuscripts at

http://www.hindawi.com
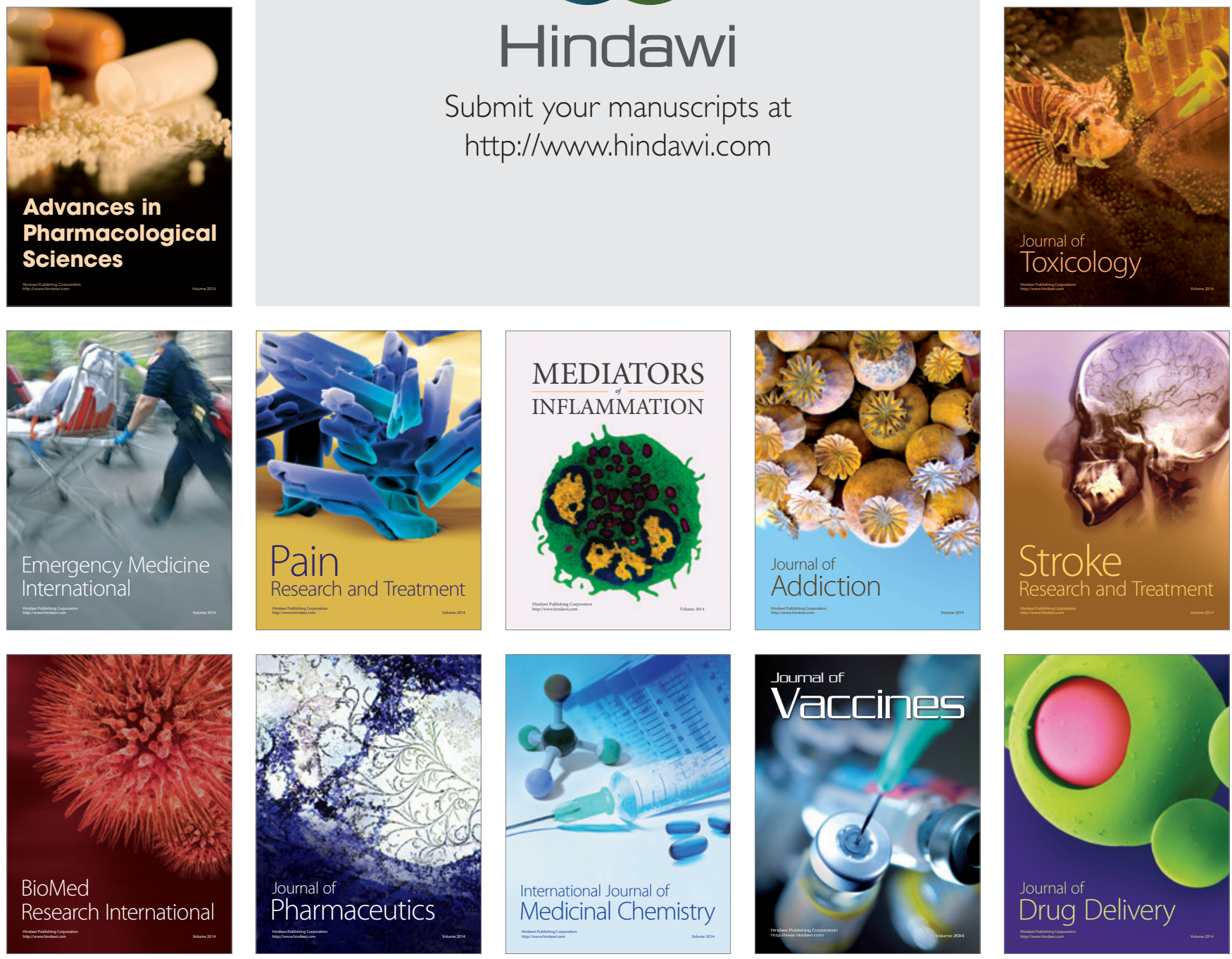